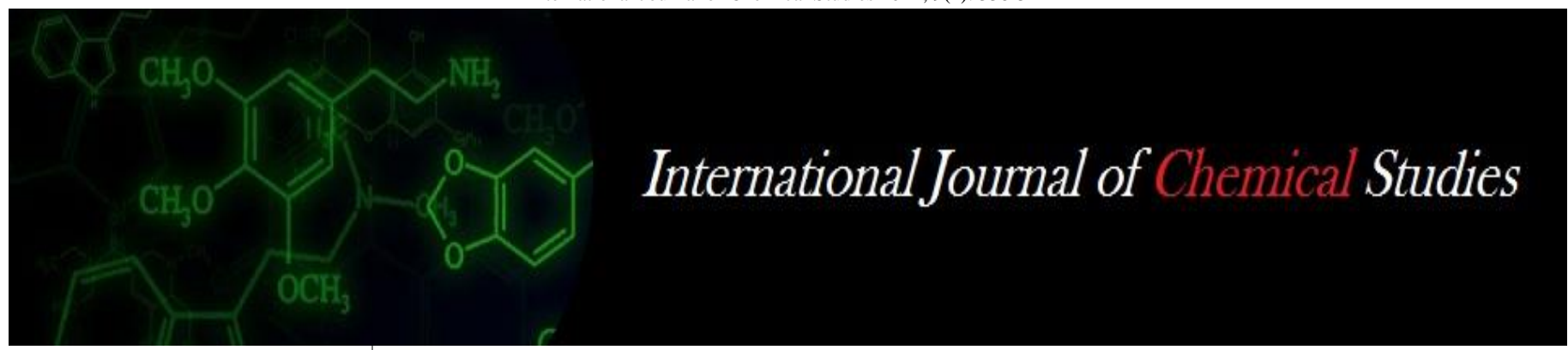

P-ISSN: 2349-8528

E-ISSN: 2321-4902

www.chemijournal.com

IJCS 2021; 9(1): 338-342

(C) 2021 IJCS

Received: 11-10-2020

Accepted: 19-11-2020

\section{Hitesh Borana}

Senior Research Fellow,

Agricultural Research Station,

Mandor, Agriculture University,

Jodhpur, Rajasthan, India

\section{Singh}

Professor, Department of

Agronomy, Agricultural

Research Station, Mandor,

Agriculture University, Jodhpur,

Rajasthan, India

\section{JR Verma}

Associate Professor, Department of Agricultural Research Station, Mandor, Agriculture University, Jodhpur, Rajasthan, India

\section{Mehriya}

Assistant Professor, Department of Agricultural Research Station, Mandor, Agriculture University, Jodhpur, Rajasthan, India

\section{UN Shukla}

Assistant Professor, Department of Agricultural Research Station, Mandor, Agriculture University, Jodhpur, Rajasthan, India

\section{Pushkar Dev}

Agricultural Research Station, Mandor, Agriculture University, Jodhpur, Rajasthan, India
Corresponding Author: Hitesh Borana

Senior Research Fellow, Agricultural Research Station, Mandor, Agriculture University, Jodhpur, Rajasthan, India

\section{Effect of weed management practices on weed dynamics in clusterbean [Cyamopsis tetragonoloba (L.) Taub]}

\author{
Hitesh Borana, I Singh, JR Verma, ML Mehriya, UN Shukla and Pushkar \\ Dev
}

DOI: $\underline{\text { htps://doi.org/10.22271/chemi.2021.v9.i1e.11250 }}$

\begin{abstract}
A field experiment was conducted to find out the effect of weed management practices on weed dynamics in clusterbean [Cyamopsis tetragonoloba (L.) Taub]. The experiment was carried out during kharif season of 2018 and ten treatment combination of different herbicides were replicated thrice in RBD. Results revealed that the early post-emergence application of imazethapyr + imazamox $50 \mathrm{~g} / \mathrm{ha}+$ $\mathrm{HW}$ at 35 DAS was found the most effective treatment which recorded lowest density of narrow and broad leaved weeds and minimum weed dry matter at all growth stages. This treatment also recorded significantly higher seed yield $(1180 \mathrm{~kg} / \mathrm{ha})$ with higher weed control efficiency $(92.8 \%)$ and lowest weed competition index $(4.6 \%)$. This practice of weed management was statistically at par with weed free season long plots and another treatment of application of imazethapyr $40 \mathrm{~g} / \mathrm{ha}$ at $20 \mathrm{DAS}+\mathrm{HW}$ at 35 DAS.
\end{abstract}

Keywords: Clusterbean, weed density, weed dry matter, early post-emergence, HW, seed yield

\section{Introduction}

Cluster bean [Cyamopsis tetragonoloba (L.) Taub] is commonly known as "Guar". The primary centre of origin of clusterbean is India (Vavilov, 1951) ${ }^{[17]}$. It is an important cash crop of rain fed areas, particularly in semi-arid and arid regions of India. This crop belongs to the family fabaceae and sub family papilionaceae. It is well adopted to the agro-climatic conditions existing in Rajasthan and is being grown in areas receiving annual rainfall from 350 to $750 \mathrm{~mm}$. Rajasthan is a major clusterbean growing state of India and it ranks first with respect to both area of 2.8 million hectares and production 1.2 million tonnes and productivity of $452 \mathrm{~kg} / \mathrm{ha}$ of clusterbean (Govt. of Rajasthan, 2020) ${ }^{[2]}$. Clusterbean is grown for different purposes viz., vegetable, green fodder, green manuring and seed. The gum/glutamin content in the endosperm of its seed (28-33 per cent) has several industrial uses viz., textiles, paper, petroleum, pharmaceuticals, food processing, cosmetics, mining explosives, oil drilling etc. thus making it a good foreign exchange earner (Kumawat et al., 2017) ${ }^{[5]}$. Tender pods of clusterbean are nutritionally wealthy in energy (116 k cal.), protein $(3.2 \mathrm{~g})$, fat (1.4 g), carbohydrate (10.8 g), vitamin-A (65.31 I.U.), vitamin-C (49 mg), calcium (57 mg) and iron $\left(4.5 \mathrm{mg}\right.$ ) per $100 \mathrm{~g}$ of edible portion (Kumar and Singh, 2002) ${ }^{[4]}$.

Weeds infects vigorously due to frequent rains and presence of weeds beyond critical period of crop-weed competition results in yield reduction to the tune of 40-45 per cent (Sangwan et al., 2016) ${ }^{[11]}$. The competition between crop and weeds caused 53.7 per cent reduction in grain yield (Singh et al., 2008) ${ }^{[14]}$. Among different weed management practices, hand weeding is traditional and efficient option but unavailability of labour at peak weeding periods and increasing labour cost oblige major limitations on economic feasibility of manual weeding (Singh and Godara, 2015) ${ }^{[13]}$. Pre-emergence application of pendimethalin is recommended in this region but if pre-emergence herbicide is skipped due to one or another reason, hence postemergence application of herbicide is effective and gives control mixed flora of narrow and broad-leaved weeds viz., Digera arvensis, Tribulus terristris, Corchorus trilocularis Phyllanthus niruri, Amaranthus viridis, Celosia argentea and Portulaca oleracea among broad-leaved weeds and Eragrostis minor, Cyperus rotundus, Cynodon dactylon and 
Dactyloctenium aegyptium among narrow leaved weeds. Keeping in view these facts, the present study was taken with an objective to find out suitable weed management practice for control of weeds in clusterbean.

\section{Materials and Methods}

The experiment was carried out at Agricultural Research Station, Mandor-Jodhpur during kharif season of 2018. The soil was loamy sand in texture, slightly alkaline in reaction ( $\mathrm{pH} 7.2)$, low in organic carbon $(0.13 \%)$ and available nitrogen $(184 \mathrm{~kg} / \mathrm{ha})$. The Field experiment was laid out in randomized block design (RBD) with ten treatments of replicated thrice. The treatments comprises of weedy check, weed free, pendimethalin $750 \mathrm{~g} / \mathrm{ha}(\mathrm{PE})$, imazethapyr $40 \mathrm{~g} / \mathrm{ha}$ at $20 \mathrm{DAS}$, pendimethalin + imazethapyr $750 \mathrm{~g} / \mathrm{ha}$ (PE), imazethapyr + imazamox $50 \mathrm{~g} / \mathrm{ha}$ at $20 \mathrm{DAS}$, pendimethalin $750 \mathrm{~g} / \mathrm{ha}(\mathrm{PE})+\mathrm{HW}$ at $25 \mathrm{DAS}$, imazethapyr $40 \mathrm{~g} / \mathrm{ha}$ at 20 DAS + HW at 35 DAS, pendimethalin + imazethapyr 750 $\mathrm{g} / \mathrm{ha}(\mathrm{PE})+\mathrm{HW}$ at $25 \mathrm{DAS}$ and imazethapyr + imazamox 50 $\mathrm{g} / \mathrm{ha}$ at $20 \mathrm{DAS}+\mathrm{HW}$ at $35 \mathrm{DAS}$. In rainy season, clusterbean variety RGC-1017 was used as a test crop in rows $30 \mathrm{~cm}$ apart using a seed rate of $15 \mathrm{~kg} / \mathrm{ha}$. Recommended dose of fertilizers, nitrogen at $10 \mathrm{~kg}$ and phosphorus at $40 \mathrm{~kg} / \mathrm{ha}$ was applied at the time of field preparation through urea and DAP fertilizers. According to treatments, pendimethalin and imazethapyr was applied as pre-mergence next day after sowing, while imazethapyr and its ready mix with imazamox was applied as early post-emergence (20 DAS). Hand weeding operation was done at 25 and 35 days after sowing as per the treatments. Weed density and weed dry matter was recorded at 40, 60, $80 \mathrm{DAS}$ and at harvest stages.

Weed control efficiency (WCE) was calculated on the basis of weed dry matter in weedy check and treated plots using formulae suggested by Mani et al. (1973) [6] while weed competition index was worked out through seed yield recorded in weed free and treated plots by using formulae given by Yadav and Mishra (1982) ${ }^{[18]}$.

\section{Results and Discussion \\ Effect on weed dynamics}

All the weed management treatments recorded significant reduction in density and weed dry matter compared to weedy check. The early post-emergence application of imazethapyr + imazamox $50 \mathrm{~g} / \mathrm{ha}$ at $20 \mathrm{DAS}+\mathrm{HW}$ at $35 \mathrm{DAS}$ and imazethapyr $40 \mathrm{~g} / \mathrm{ha}$ at $20 \mathrm{DAS}+\mathrm{HW}$ at $35 \mathrm{DAS}$ significantly reduced the density and dry matter of narrow and broad leaved weeds as compared to weedy check plots and other treatments at 40, 60, 80 DAS and at harvest during experimentation (Table 1 and 2). It was due to effective control of weeds by early post-emergence application of imazethapyr + imazamox (ready mix) which was found effective against all kind of weeds especially broad-leaved weeds and resulted in significant reduction in the population of weeds. Similar results are reported by Gupta et al. (2016) and Sharma et al. (2017) ${ }^{[12]}$ in clusterbean crop. Punia et al. (2011) also reported better control of weeds in clusterbean by imazethapyr. Further, pre emergence application of pendimethalin + imazethapyr $750 \mathrm{~g} / \mathrm{ha}+\mathrm{HW}$ at $25 \mathrm{DAS}$ and pendimethalin $750 \mathrm{~g} / \mathrm{ha}(\mathrm{PE})+\mathrm{HW}$ at 25 DAS recorded significantly lower density and weed dry matter compared to weedy check and alone application of these herbicides but these treatments were statistically at par with alone application of imazethapyr + imazamox $50 \mathrm{~g} / \mathrm{ha}$ at $20 \mathrm{DAS}$. A judicious combination of chemical and cultural methods of weed management would not only reduce the expenditure on herbicides but would benefit the crop by providing proper aeration and conservation of moisture (Prakash et al., 1991) ${ }^{[8]}$. The weed control efficiency showed the efficacy of herbicides with respect to controlling weeds over weedy check. Data (Table 3) revealed that higher weed control efficiency brought up by the early post-emergence application of imazethapyr + imazamox $50 \mathrm{~g} / \mathrm{ha}$ at $20 \mathrm{DAS}+\mathrm{HW}$ at $35 \mathrm{DAS}$ followed by imazethapyr $40 \mathrm{~g} / \mathrm{ha}$ at $20 \mathrm{DAS}+1 \mathrm{HW}$ at $35 \mathrm{DAS}$ and attained highest weed control efficiency viz., 92.8 and 91.1 per cent, respectively. However, the minimum weed control efficiency $(61.6 \%)$ was recorded under pre emergence application of pendimethalin $750 \mathrm{~g} / \mathrm{ha}$. Herbicide imazethapyr has a broad spectrum control of weeds (Saltoni et al., 2004) [10]. Herbicide imazethapyr inhibits the plastid aceto-lactate synthesis (ALS) in plants. The ALS inhibitors stop cell division and reduces carbohydrate translocation in susceptible plants (Gupta, 2008) ${ }^{[3]}$. Imazethapyr by virtue of wide spectrum weed control (both grassy and broad-leaved weeds) throughout the growing season without causing any phytotoxicity to crop plants emerged as a technically sound and economically viable option of post-emergence application in this study. Similar results are reported by Patil et al. (2013) [7] and Sharma et al. (2017) [12] in clusterbean crop. Application of imazethapyr + imazamox $50 \mathrm{~g} / \mathrm{ha}$ (ready mix) at 20 DAS without hand weeding recorded weed control efficiency of 74 per cent which was further improved up to 92.8 per cent when this treatment was super imposed with hand weeding at 35 DAS. The seed yield and weed control efficiency was positively correlated with correlation coefficient of 0.956. This was further supported by the regression analysis, which revealed that as the density and dry weight of weeds decreased, the seed yield of clusterbean was increased by $10.52 \mathrm{~kg} / \mathrm{ha}$ (Figure 1) in terms of weed control efficiency.

Weed index indicates the loss of yield caused by weed-crop competition under particular treatment in comparison to yield obtained from weed free plot. Application of imazethapyr + imazamox $50 \mathrm{~g} / \mathrm{ha}$ (ready mix) at $20 \mathrm{DAS}+\mathrm{HW}$ at $35 \mathrm{DAS}$ recorded lowest weed index (4.6) followed by imazethapyr 40 $\mathrm{g} / \mathrm{ha}$ at $20 \mathrm{DAS}+\mathrm{HW}$ at 35 DAS (9.4) (Table 3). It was due to lowest weed density and weed dry matter recorded in these treatments which ultimately provided weed free environment and produced higher yield attributes and yield. The huge losses in yield was observed in weedy check plots due to heavy flushes of weeds during entire crop growth period and thereby caused severe weed-crop competition by the uncontrolled weed growth and thus, resulted in higher yield losses. These results corroborate the findings of Sharma et al. (2017) ${ }^{[12]}$ and Singh and Godara (2015) ${ }^{[13]}$ in cluster bean crop. The seed yield and weed index was negatively correlated with correlation co-efficient of 1.00 . This was further supported by the regression analysis, which revealed that as the density and dry weight of weeds increased, the seed yield of clusterbean was decreased by $12.37 \mathrm{~kg} / \mathrm{ha}$ (Figure 2) in terms of weed index. 
Table 1: Density of weeds as influenced by various weed management treatments

\begin{tabular}{|c|c|c|c|c|c|c|c|c|}
\hline \multirow{3}{*}{ Treatments } & \multicolumn{8}{|c|}{ Weed density $\left(\mathrm{No} . \mathrm{m}^{2}\right)$} \\
\hline & \multicolumn{2}{|c|}{40 DAS } & \multicolumn{2}{|c|}{ 60 DAS } & \multicolumn{2}{|c|}{80 DAS } & \multicolumn{2}{|c|}{ At harvest } \\
\hline & NLW & BLW & NLW & BLW & NLW & BLW & NLW & BLW \\
\hline Weedy check & $\begin{array}{c}2.91 \\
\left(8.00^{*}\right)\end{array}$ & $\begin{array}{c}10.22 \\
(104.0)\end{array}$ & $\begin{array}{c}3.62 \\
(12.67)\end{array}$ & $\begin{array}{c}11.98 \\
(143.00)\end{array}$ & $\begin{array}{c}3.08 \\
(9.00)\end{array}$ & $\begin{array}{c}10.98 \\
(120.00)\end{array}$ & $\begin{array}{c}2.61 \\
(6.33) \\
\end{array}$ & $\begin{array}{c}9.62 \\
(92.00)\end{array}$ \\
\hline Weed free & $0.71(0.00)$ & $0.71(0.00)$ & $0.71(0.00)$ & $0.71(0.00)$ & $\begin{array}{c}0.71 \\
(0.00)\end{array}$ & $0.71(0.00)$ & $\begin{array}{c}0.71 \\
(0.00)\end{array}$ & $\begin{array}{c}0.71 \\
(0.00)\end{array}$ \\
\hline Pendimethalin $750 \mathrm{~g} / \mathrm{ha}(\mathrm{PE})$ & $2.55(6.00)$ & $\begin{array}{c}5.59 \\
(31.00)\end{array}$ & $2.86(7.67)$ & $6.36(40.33)$ & $\begin{array}{c}2.68 \\
(6.67)\end{array}$ & $5.86(34.33)$ & $\begin{array}{c}2.20 \\
(4.33)\end{array}$ & $\begin{array}{c}5.52 \\
(30.33)\end{array}$ \\
\hline Imazethapyr $40 \mathrm{~g} / \mathrm{ha}$ at $20 \mathrm{DAS}$ & $2.48(5.67)$ & $\begin{array}{c}5.39 \\
(28.67)\end{array}$ & $2.80(7.33)$ & $5.94(35.00)$ & $\begin{array}{c}2.61 \\
(6.33)\end{array}$ & $5.58(31.00)$ & $\begin{array}{c}2.12 \\
(4.00)\end{array}$ & $\begin{array}{c}5.23 \\
(27.00)\end{array}$ \\
\hline Pendimethalin + imazethapyr $750 \mathrm{~g} / \mathrm{ha}(\mathrm{PE})$ & $2.48(5.67)$ & $\begin{array}{c}5.31 \\
(28.00) \\
\end{array}$ & $2.74(7.00)$ & $5.68(32.00)$ & $\begin{array}{c}2.55 \\
(6.00)\end{array}$ & $5.52(30.00)$ & $\begin{array}{c}2.04 \\
(3.67)\end{array}$ & $\begin{array}{c}5.21 \\
(26.67)\end{array}$ \\
\hline Imazethapyr + imazamox $50 \mathrm{~g} / \mathrm{ha}$ at $20 \mathrm{DAS}$ & $2.20(4.33)$ & $\begin{array}{c}3.52 \\
(12.33) \\
\end{array}$ & $2.26(4.67)$ & $4.33(18.67)$ & $\begin{array}{c}1.95 \\
(3.33) \\
\end{array}$ & $4.10(16.33)$ & $\begin{array}{c}1.87 \\
(3.00) \\
\end{array}$ & $\begin{array}{c}3.52 \\
(12.00) \\
\end{array}$ \\
\hline Pendimethalin $750 \mathrm{~g} / \mathrm{ha}(\mathrm{PE})+\mathrm{HW}$ at $25 \mathrm{DAS}$ & $2.12(4.00)$ & $\begin{array}{c}3.94 \\
(15.33) \\
\end{array}$ & $2.12(4.00)$ & $3.85(14.67)$ & $\begin{array}{c}1.96 \\
(3.33)\end{array}$ & $3.81(14.00)$ & $\begin{array}{c}1.68 \\
(2.33)\end{array}$ & $\begin{array}{c}3.76 \\
(13.67)\end{array}$ \\
\hline Imazethapyr $40 \mathrm{~g} / \mathrm{ha}$ at $20 \mathrm{DAS}+\mathrm{HW}$ at $35 \mathrm{DAS}$ & $1.68(2.33)$ & $2.39(5.33)$ & $1.56(2.00)$ & $2.32(5.00)$ & $\begin{array}{c}1.47 \\
(1.67)\end{array}$ & $2.27(4.67)$ & $\begin{array}{c}1.22 \\
(1.00)\end{array}$ & $2.27(4.67)$ \\
\hline $\begin{array}{c}\text { Pendimethalin + imazethapyr } 750 \mathrm{~g} / \mathrm{ha}(\mathrm{PE})+\mathrm{HW} \\
\text { at 25 DAS }\end{array}$ & $2.12(4.00)$ & $\begin{array}{c}4.06 \\
(16.33)\end{array}$ & $2.03(3.67)$ & $3.54(12.33)$ & $\begin{array}{l}1.77 \\
(2.67)\end{array}$ & $3.39(11.00)$ & $\begin{array}{c}1.58 \\
(2.00)\end{array}$ & $\begin{array}{c}3.24 \\
(10.00)\end{array}$ \\
\hline $\begin{array}{c}\text { Imazethapyr + imazamox } 50 \mathrm{~g} / \mathrm{ha} \text { at } 20 \mathrm{DAS}+\mathrm{HW} \\
\text { at } 35 \mathrm{DAS}\end{array}$ & $1.58(2.00)$ & $2.33(5.00)$ & $1.34(1.33)$ & $2.14(4.33)$ & $\begin{array}{c}1.22 \\
(1.00) \\
\end{array}$ & $2.12(4.00)$ & $\begin{array}{l}1.08 \\
(0.67) \\
\end{array}$ & $2.04(3.67)$ \\
\hline S Em \pm & 0.07 & 0.32 & 0.10 & 0.32 & 0.06 & 0.20 & 0.06 & 0.18 \\
\hline $\mathrm{CD}(\mathrm{P}=0.05)$ & 0.22 & 0.96 & 0.32 & 0.95 & 0.20 & 0.60 & 0.18 & 0.55 \\
\hline
\end{tabular}

*Figures in parentheses are the original value

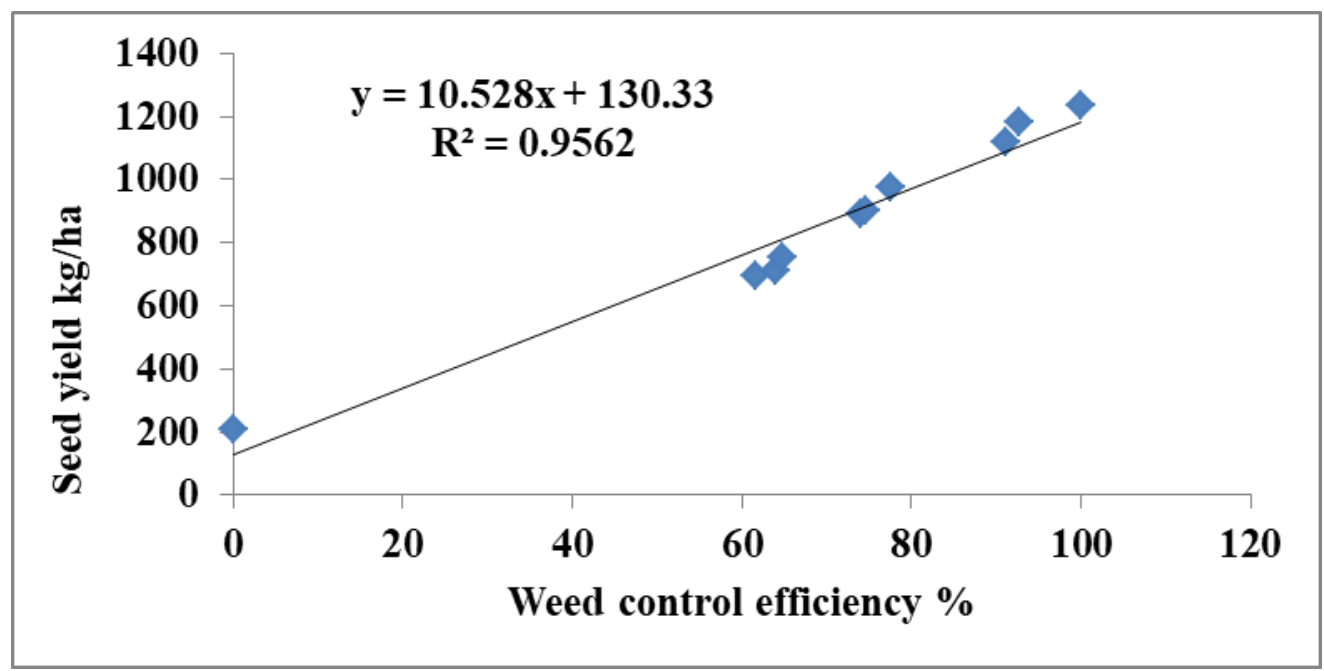

Fig 1: Regression analysis between seed yield and weed control efficiency of clusterbean

Table 2: Dry matter of weeds as influenced by various weed management treatments

\begin{tabular}{|c|c|c|c|c|c|c|c|c|c|c|c|c|}
\hline \multirow{3}{*}{ Treatments } & \multicolumn{12}{|c|}{ Dry matter of weeds $\left(\mathrm{g} / \mathrm{m}^{2}\right)$} \\
\hline & \multicolumn{3}{|c|}{40 DAS } & \multicolumn{3}{|c|}{60 DAS } & \multicolumn{3}{|c|}{$\begin{array}{r}\text { 80 DAS } \\
\end{array}$} & \multicolumn{3}{|c|}{ At harvest } \\
\hline & NLW & BLW & Total & NLW & BLW & Total & NLW & BLW & Total & NLW & BLW & Total \\
\hline & $\begin{array}{c}1.47 \\
(1.67 *)\end{array}$ & $\begin{array}{c}4.91 \\
(19.67)\end{array}$ & $\begin{array}{c}4.67 \\
(21.33)\end{array}$ & $\begin{array}{c}1.94 \\
(3.27)\end{array}$ & $\begin{array}{c}8.62 \\
(74.13)\end{array}$ & $\begin{array}{c}8.81 \\
(77.40)\end{array}$ & \begin{tabular}{|c|}
2.53 \\
$(5.90)$ \\
\end{tabular} & $\begin{array}{c}12.91 \\
(166.67)\end{array}$ & $\begin{array}{c}13.14 \\
(172.57)\end{array}$ & $\begin{array}{c}2.86 \\
(7.71) \\
\end{array}$ & $\begin{array}{c}14.28 \\
(203.33)\end{array}$ & $\begin{array}{c}14.54 \\
(211.04)\end{array}$ \\
\hline & $\begin{array}{c}0.71 \\
(0.00)\end{array}$ & $\begin{array}{c}0.71 \\
(0.00)\end{array}$ & & $\begin{array}{c}0.7 \\
(0.00)\end{array}$ & & $\begin{array}{c}0.71 \\
(0.00)\end{array}$ & $\begin{array}{c}0.71 \\
(0.00)\end{array}$ & & & $\begin{array}{c}0.71 \\
(0.00)\end{array}$ & & \\
\hline $\begin{array}{r}\text { Pendimetha } \\
\text { (P }\end{array}$ & $\begin{array}{c}1.33 \\
(1.28)\end{array}$ & $\begin{array}{c}2.96 \\
(8.25)\end{array}$ & $\begin{array}{c}3.17 \\
(9.53)\end{array}$ & \begin{tabular}{|c|}
1.66 \\
$(2.27)$
\end{tabular} & $\begin{array}{c}5.28 \\
(27.33)\end{array}$ & $\begin{array}{c}5.49 \\
(29.60)\end{array}$ & $\begin{array}{c}2.12 \\
(4.05) \\
\end{array}$ & $\begin{array}{c}8.00 \\
(63.53)\end{array}$ & $\begin{array}{r}8 . \\
(67\end{array}$ & $\begin{array}{c}2.46 \\
(5.64)\end{array}$ & $\begin{array}{r}8 . \\
(75\end{array}$ & $\begin{array}{c}8.99 \\
(81.00)\end{array}$ \\
\hline $\begin{array}{r}\text { Imazethapyr at } \\
20 \mathrm{DA} \\
\end{array}$ & $\begin{array}{c}1.26 \\
(1.08) \\
\end{array}$ & $\begin{array}{c}2.91 \\
(7.96) \\
\end{array}$ & $\begin{array}{c}3.09 \\
(9.04) \\
\end{array}$ & \begin{tabular}{|c|}
1.66 \\
$(2.24)$ \\
\end{tabular} & $\begin{array}{c}5.17 \\
(26.27) \\
\end{array}$ & $\begin{array}{c}5.39 \\
(28.51) \\
\end{array}$ & \begin{tabular}{|c|}
2.15 \\
$(4.12)$ \\
\end{tabular} & $\begin{array}{c}7.78 \\
(60.00) \\
\end{array}$ & $\begin{array}{r}8.1 \\
(64 . \\
\end{array}$ & $\begin{array}{c}2.45 \\
(5.52) \\
\end{array}$ & $\begin{array}{r}8 . \\
(70 . \\
\end{array}$ & $\begin{array}{c}8.75 \\
(76.04) \\
\end{array}$ \\
\hline $\begin{array}{c}\text { Pendimethalin }+ \\
\text { imazethapyr } 750 \mathrm{~g} / \mathrm{ha} \\
\text { (PE) }\end{array}$ & $\begin{array}{c}1.23 \\
(1.03)\end{array}$ & $\begin{array}{c}2.84 \\
(7.59)\end{array}$ & $\begin{array}{c}3.02 \\
(8.62)\end{array}$ & $\begin{array}{c}1.64 \\
(2.20)\end{array}$ & $\begin{array}{c}5.02 \\
(24.73)\end{array}$ & $\begin{array}{c}5.24 \\
(26.93)\end{array}$ & \begin{tabular}{|c|}
2.17 \\
$(4.21)$
\end{tabular} & $\begin{array}{c}7.75 \\
(59.51)\end{array}$ & $\begin{array}{c}8.01 \\
(63.73)\end{array}$ & $\begin{array}{c}2.44 \\
(5.43)\end{array}$ & $\begin{array}{c}8.33 \\
(68.97)\end{array}$ & $\begin{array}{c}8.65 \\
(74.40)\end{array}$ \\
\hline $\begin{array}{c}\text { Imazethapyr + imazamox } \\
50 \mathrm{~g} / \mathrm{ha} \text { at } 20 \mathrm{DAS}\end{array}$ & $\begin{array}{c}1.12 \\
(0.76)\end{array}$ & $\begin{array}{c}2.27 \\
(4.64) \\
\end{array}$ & $\begin{array}{c}2.43 \\
(5.40) \\
\end{array}$ & $\begin{array}{c}1.38 \\
(1.42) \\
\end{array}$ & $\begin{array}{c}4.39 \\
(18.79) \\
\end{array}$ & $\begin{array}{c}4.55 \\
(20.21) \\
\end{array}$ & $\begin{array}{c}1.99 \\
(3.26) \\
\end{array}$ & $\begin{array}{c}6.38 \\
(40.18) \\
\end{array}$ & $\begin{array}{c}6.63 \\
(43.44) \\
\end{array}$ & $\begin{array}{c}1.97 \\
(3.37) \\
\end{array}$ & $\begin{array}{c}7.21 \\
(51.55) \\
\end{array}$ & $\begin{array}{c}7.44 \\
(54.92) \\
\end{array}$ \\
\hline $\begin{array}{l}\text { Pendimethalin } 750 \mathrm{~g} / \mathrm{ha} \\
(\mathrm{PE})+\mathrm{HW} \text { at } 25 \mathrm{DAS}\end{array}$ & $\begin{array}{c}1.10 \\
(0.72)\end{array}$ & $\begin{array}{c}2.23 \\
(4.45)\end{array}$ & $\begin{array}{c}2.38 \\
(5.18)\end{array}$ & \begin{tabular}{|c|}
1.37 \\
$(1.37)$
\end{tabular} & $\begin{array}{c}4.24 \\
(17.46)\end{array}$ & $\begin{array}{c}4.40 \\
(18.83)\end{array}$ & \begin{tabular}{|c|}
1.89 \\
$(3.08)$
\end{tabular} & $\begin{array}{c}6.30 \\
(39.17)\end{array}$ & $\begin{array}{c}6.54 \\
(42.25)\end{array}$ & \begin{tabular}{|c|}
1.93 \\
$(3.21)$
\end{tabular} & $\begin{array}{c}7.11 \\
(50.06)\end{array}$ & $\begin{array}{c}7.33 \\
(53.27)\end{array}$ \\
\hline
\end{tabular}




\begin{tabular}{|c|c|c|c|c|c|c|c|c|c|c|c|c|}
\hline $\begin{array}{c}\text { Imazethapyr } 40 \mathrm{~g} / \mathrm{ha} \text { at } 20 \\
\text { DAS + HW at } 35 \text { DAS }\end{array}$ & $\begin{array}{r}0.87 \\
(0.26) \\
\end{array}$ & $\begin{array}{c}1.48 \\
(1.70)\end{array}$ & $\begin{array}{c}1.57 \\
(1.96) \\
\end{array}$ & $\begin{array}{r}1.02 \\
(0.54) \\
\end{array}$ & $\begin{array}{c}2.44 \\
(5.46) \\
\end{array}$ & $\begin{array}{c}2.55 \\
(6.00) \\
\end{array}$ & \begin{tabular}{|l}
1.14 \\
$(0.80)$ \\
\end{tabular} & $\begin{array}{c}3.32 \\
(10.53) \\
\end{array}$ & $\begin{array}{c}3.44 \\
(11.33) \\
\end{array}$ & $\begin{array}{c}1.29 \\
(1.17) \\
\end{array}$ & $\begin{array}{c}4.26 \\
(17.67) \\
\end{array}$ & $\begin{array}{c}4.40 \\
(18.84) \\
\end{array}$ \\
\hline $\begin{array}{c}\text { Pendimethalin }+ \\
\text { imazethapyr } 750 \mathrm{~g} / \mathrm{ha} \\
\text { (PE) + HW at } 25 \text { DAS }\end{array}$ & $\begin{array}{l}1.02 \\
(0.55)\end{array}$ & $\begin{array}{c}2.16 \\
(4.16)\end{array}$ & $\begin{array}{l}2.28 \\
(4.70)\end{array}$ & $\begin{array}{c}1.27 \\
(1.12)\end{array}$ & $\begin{array}{c}3.99 \\
(15.44)\end{array}$ & $\begin{array}{c}4.13 \\
(16.56)\end{array}$ & $\begin{array}{c}1.82 \\
(2.81)\end{array}$ & $\begin{array}{c}6.02 \\
(35.74)\end{array}$ & $\begin{array}{c}6.64 \\
(38.55)\end{array}$ & $\begin{array}{c}1.81 \\
(2.77)\end{array}$ & $\begin{array}{c}6.71 \\
(44.49)\end{array}$ & $\begin{array}{c}6.91 \\
(47.26)\end{array}$ \\
\hline $\begin{array}{c}\text { Imazethapyr + imazamox } \\
50 \mathrm{~g} / \mathrm{ha} \text { at } 20 \mathrm{DAS}+\mathrm{HW} \\
\text { at } 35 \mathrm{DAS}\end{array}$ & $\begin{array}{c}0.84 \\
(0.20)\end{array}$ & $\begin{array}{c}1.34 \\
(1.29)\end{array}$ & $\begin{array}{c}1.41 \\
(1.59)\end{array}$ & $\begin{array}{c}0.97 \\
(0.44)\end{array}$ & $\begin{array}{c}2.25 \\
(4.56)\end{array}$ & $\begin{array}{c}2.34 \\
(5.00)\end{array}$ & $\begin{array}{l}1.07 \\
(0.65)\end{array}$ & $\begin{array}{l}3.14 \\
(9.35)\end{array}$ & $\begin{array}{c}3.24 \\
(10.00)\end{array}$ & $\begin{array}{c}1.26 \\
(1.10)\end{array}$ & $\begin{array}{c}3.82 \\
(14.08)\end{array}$ & $\begin{array}{c}3.96 \\
(15.18)\end{array}$ \\
\hline $\begin{array}{c}\mathrm{S} \mathrm{Em} \pm \\
\mathrm{CD}(\mathrm{P}=0.05)\end{array}$ & $\begin{array}{l}0.03 \\
0.10 \\
\end{array}$ & $\begin{array}{l}0.06 \\
0.20\end{array}$ & $\begin{array}{l}0.06 \\
0.20\end{array}$ & $\begin{array}{l}0.05 \\
0.15\end{array}$ & $\begin{array}{l}0.14 \\
0.42\end{array}$ & $\begin{array}{l}0.13 \\
0.40\end{array}$ & $\begin{array}{l}0.06 \\
0.19\end{array}$ & $\begin{array}{l}0.15 \\
0.47\end{array}$ & $\begin{array}{l}0.15 \\
0.46\end{array}$ & $\begin{array}{l}0.07 \\
0.21\end{array}$ & $\begin{array}{l}0.24 \\
0.53\end{array}$ & $\begin{array}{l}0.18 \\
0.54\end{array}$ \\
\hline
\end{tabular}

*Figures in parentheses are the original value

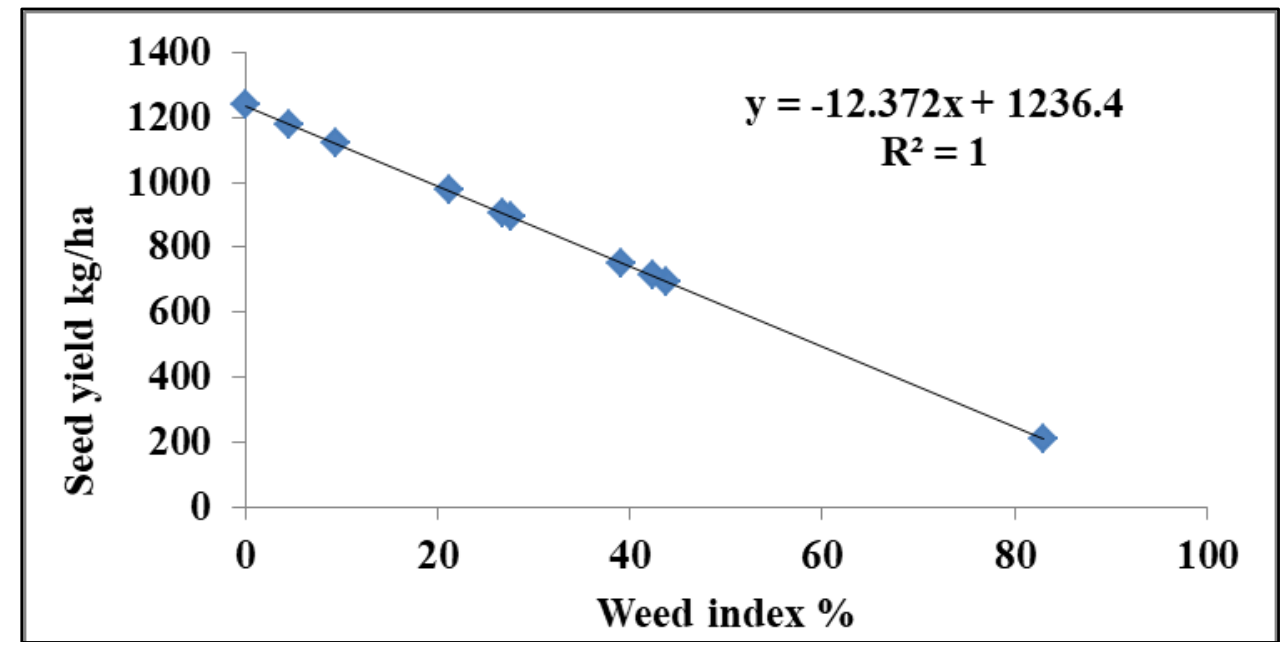

Fig 2: Regression analysis between seed yield and weed index of clusterbean

Table 3: Weed control efficiency, weed index and seed yield as influenced by various weed management treatments

\begin{tabular}{|c|c|c|c|c|}
\hline Treatments & WCE (\%) & Weed index $(\%)$ & Seed yield (kg/ha) & Net return ( $\square /$ ha) \\
\hline Weedy check & 0.00 & 83.0 & 210.0 & $-9,333$ \\
\hline Weed free & 100.00 & 0.00 & 1237.0 & 47,663 \\
\hline Pendimethalin $750 \mathrm{~g} / \mathrm{ha}(\mathrm{PE})$ & 61.6 & 43.8 & 694.0 & 20,720 \\
\hline Imazethapyr $40 \mathrm{~g} / \mathrm{ha}$ at $20 \mathrm{DAS}$ & 63.9 & 42.4 & 713.0 & 22,819 \\
\hline Pendimethalin + imazethapyr $750 \mathrm{~g} / \mathrm{ha}(\mathrm{PE})$ & 64.7 & 39.1 & 752.0 & 24,076 \\
\hline Imazethapyr + imazamox $50 \mathrm{~g} / \mathrm{ha}$ at $20 \mathrm{DAS}$ & 74.0 & 27.7 & 893.0 & 34,370 \\
\hline Pendimethalin $750 \mathrm{~g} / \mathrm{ha}(\mathrm{PE})+\mathrm{HW}$ at $25 \mathrm{DAS}$ & 74.7 & 26.8 & 903.0 & 32,275 \\
\hline Imazethapyr $40 \mathrm{~g} / \mathrm{ha}$ at $20 \mathrm{DAS}+\mathrm{HW}$ at $35 \mathrm{DAS}$ & 91.1 & 9.4 & 1120.0 & 46,002 \\
\hline Pendimethalin + imazethapyr $750 \mathrm{~g} / \mathrm{ha}(\mathrm{PE})+\mathrm{HW}$ at $25 \mathrm{DAS}$ & 77.6 & 21.2 & 975.0 & 36,619 \\
\hline Imazethapyr + imazamox $50 \mathrm{~g} / \mathrm{ha}$ at $20 \mathrm{DAS}+\mathrm{HW}$ at $35 \mathrm{DAS}$ & 92.8 & 4.6 & 1180 & 49,693 \\
\hline $\begin{array}{c}\mathrm{S} \mathrm{Em} \pm \\
\mathrm{CD}(\mathrm{P}=0.05)\end{array}$ & - & - & $\begin{array}{c}44.23 \\
131.44\end{array}$ & - \\
\hline
\end{tabular}

\section{Effect on seed yield}

Among various herbicidal treatments, significantly higher seed yield $(1180 \mathrm{~kg} / \mathrm{ha})$ was recorded with the application of imazethapyr + imazamox $50 \mathrm{~g} / \mathrm{ha}$ at 20 DAS but it was found equally effective with imazethapyr $40 \mathrm{~g} / \mathrm{ha}$ at $20 \mathrm{DAS}+\mathrm{HW}$ at 35 DAS $(1120 \mathrm{~kg} / \mathrm{ha})$. Both these treatments were significantly superior over rest of the treatments and remained statistically at par with weed free season long plot. Application of pendimethalin + imazethapyr $750 \mathrm{~g} / \mathrm{ha}+\mathrm{HW}$ at $25 \mathrm{DAS}$ and imazethapyr $40 \mathrm{~g} / \mathrm{ha}$ at $20 \mathrm{DAS}+\mathrm{HW}$ at 35 DAS recorded increase of 765 and $910 \mathrm{~kg} / \mathrm{ha}$ higher seed yield, respectively over weedy check. The seed yield was reduced by 24.3 per cent when application of imazethapyr + imazamox $50 \mathrm{~g} / \mathrm{ha}$ at 20 DAS was applied without one hand weeding at 35 DAS. Application of imazethapyr + imazamox $50 \mathrm{~g} / \mathrm{ha}$ at 20 DAS without hand weeding produce seed yield of $893 \mathrm{~kg} / \mathrm{ha}$ which was further improved by $287 \mathrm{~kg} / \mathrm{ha}$ when this treatment was super imposed with hand weeding at 35 DAS. This might be due to better control of weeds as herbicides were applied 20 DAS and most of the weeds were suppressed at 2-3 leaf stage. But even then some of remaining weeds started flourishing and started competition with the crop plants during the critical phase of crop-weed competition. All those weeds were suppressed when these treatments were super imposed with hand weeding 35 DAS and due to this a complete/full weed free period existed during the entire crop pendency. These results corroborate the findings of Gupta et al. (2016), Sharma et al. (2017) ${ }^{[12]}$ and Yadav and Mundra (2017) ${ }^{[19]}$ in clusterbean.

\section{Conclusion}

It can be concluded that early post-emergence application of imazethapyr + imazamox $50 \mathrm{~g} / \mathrm{ha}$ at $20 \mathrm{DAS}+\mathrm{HW}$ at $35 \mathrm{DAS}$ was more effective in management of both narrow and broad leaved weeds resulting higher grain yield and economics of clusterbean (1180 kg/ha and $\square$ 49,693/ha, respectively).

\section{References}

1. Gomez KA, Gomez AA. Statistical procedures for agricultural research, John Wiley (II Edition). New York 1984,680p. 
2. Government of Rajasthan. Crop-wise Fourth Advance Estimates. Commissionerate of Agriculture, RajasthanJaipur 2020.

3. Gupta OP. Functional features of some currently used herbicides. Modern Weed Management 3rd Revised Edition, AGROBIOS (INDIA), Jodhpur 2008,223p.

4. Kumar D, Singh NB. Guar in India. Scientific pubishers, Jodhpur, India 2002.

5. Kumawat P, Kaushik MK, Meena VK, Chouhan BS, Meena RK, Kumar R. Effect of weed management and fertility levels on productivity of clusterbean [Cyamopsis tetragonoloba (L.) Taub]. Legume Research 2017;40(5):884-889.

6. Mani VS, Malla MC, Gautam KC, Bhagwandas. Weed Killing chemicals in potato cultivars. Indian Farming 1973;32(8):17-18.

7. Patil BT, Bhalekar MN, Shinde KG. Weed management in clusterbean (Cyamopsis tetragonoloba L.). National Symposium on Abiotic and Biotic stress management in vegetable crops 2013.

8. Prakash V, Prasad K, Singh P. Chemical weed control in soybean. Indian Journal of Weed Science 1991;23(1\&2):2931.

9. Puniya SS, Singh S, Yadav D. Bioefficacy of imazethapyr and chlorimuron-ethyl in clusterbean and their residual effect on succeeding rabi crops. Indian Journal of Weed Science 2011;43(1 \& 2):48-53.

10. Saltoni N, Shropshire C, Cowan T, Sikkema P. Tolerance of black beans (Phaseolus vulgaris) to soil application of S-metalochlor and imazethapyr. Weed Technology 2004;18:111-118.

11. Sangwan M, Singh S, Satyavan. Efficacy of sequential application of imazethapyr + imazamox and propaquizafop in clusterbean (Cyamopsis tetragonoloba) in two texturally different soils. Indian Journal of Agronomy 2016;61:519-52.

12. Sharma K, Rawat GS, Gaur D, Sharma A. Effect of postemergence herbicides on weed control, growth and yield of clusterbean [Cyamopsis tetragonoloba (L.) Taub] in M.P. Agricultural Science Digest 2017;37(3):179-184.

13. Singh R, Godara AS. Weed control efficiency of postemergence herbicides and their effect on productivity of clusterbean [Cyamopsis tetragonoloba (L.) Taub]. Legume Research 2015;38(3):415-418.

14. Singh SK, Jain AK, Puniya BL. Integrated weed management in clusterbean [Cyamopsis tetragonoloba (L.)]. Indian Journal of Agricultural Sciences 2008;70(2):850-852.

15. Gupta V, Singh SP, Yadav RS. Yield performance and nutrient uptake as influenced by integrated weed management in clusterbean. Indian Journal of Weed Science 2015;47(1):82-84.

16. Singh SP, Yadav RS, Sharma V. Weed control in clusterbean through post-emergence herbicides. Indian Journal of Weed Science 2016;48(2):202-205.

17. Vavilov NI. The origin, variation, immunity and breeding of cultivated plants. Chronic Botanica 1951;13:1-54.

18. Yadav JP, Mishra MRC. Naveen Prayogic Krishi. A hand book of Agriculture. Kanti Prakashan, Etawah 1982.

19. Yadav RK, Mundra SL. Weed management and sulphur nutrition in clusterbean for higher productivity and profitability. Journal of Pharmacognosy and Phytochemistry 2017;6(3):06-08. 